\title{
Recollection and familiarity in aging individuals: Gaining insight into relationships with medial temporal lobe structural integrity
}

\author{
Dorothee Schoemaker ${ }^{1}$ \\ Charlotte Mascret ${ }^{1}$ \\ D. Louis Collins $\mathbf{s}^{2,3}$ \\ Elsa $\mathrm{Yu}^{4}$ \\ Serge Gauthier ${ }^{1} \quad$ Jens C. Pruessner ${ }^{1,2,5}$
}

${ }^{1}$ McGill University Research Center for Studies in Aging, Montreal, Quebec, Canada

${ }^{2}$ Department of Neurology/Neurosurgery, McGill University, Montreal, Quebec, Canada

${ }^{3}$ McConnell Brain Imaging Centre, Montreal Neurological Institute, Montreal, Quebec, Canada

${ }^{4}$ Douglas Mental Health University Institute, Montreal, Quebec, Canada

${ }^{5}$ Department of Psychology, University of Constance, Constance, Germany

\section{Correspondence}

Dorothee Schoemaker, McGill University Research Center for Studies in Aging, Montreal, Quebec, Canada.

Email: dorothee.schoemaker@mail.mcgill.ca

\section{Funding information}

This work was supported by a research grant "Maladie d'Alzheimer et les Maladies Apparentées" jointly sponsored by the Agence Nationale de la Recherche (ANR), Fonds de Recherche du Québec - Santé (FRQS), and the Canadian Institutes of Health Research (CIHR) (grant 23638). DS doctoral training is funded by an FRQS doctoral award (grant 29793). JCP is supported by an FRQS Chercheur National salary award (grant 22507).

\begin{abstract}
Dual-process theories posit that two separate processes are involved in recognition, namely recollection and familiarity. Studies investigating the neuroanatomical substrates of these two processes have frequently revealed that, while recollection is functionally linked with the hippocampus, familiarity appears to be associated with perirhinal and/or entorhinal cortices integrity. Interestingly these regions are known to be sensitive to normal and neuropathological aging processes. The objective of this study was to examine the effects of aging on recollection and familiarity performance, as well as to investigate associations with the rate of false alarms. In older individuals, we further aimed to explore relationships between these recognition variables and structural integrity of the hippocampus and the entorhinal and perirhinal cortices. Younger $(N=56)$ and older $(N=59)$ adults were tested on a computerized recollection and familiarity task. In a separate session, older adults $(N=56)$ underwent a structural MRI. Hippocampal, entorhinal and perihinal cortices volumes were automatically segmented and then manually corrected to ensure validity of the volumetric assessment. Regional volumes were normalized for total intracranial volume. While the overall recognition performance did not significantly differ across groups, our results reveal a decrease in recollection, together with an increase in familiarity in older adults. The increase reliance on familiarity was significantly and positively associated with the rate of false alarms. In the older adult sample, significant positive associations were found between recollection estimates and normalized hippocampal volumes. The normalized total hippocampal volume accounted for $25 \%$ of the variance in recollection performance. No correlation was found between any recognition variables and perirhinal or entorhinal cortices volumes. Overall, our results suggest that the age-related impairment in recollection is linked with reduced hippocampal structural integrity.
\end{abstract}

\section{KEYWORDS}

aging, recollection, familiarity, structural neuroimaging

\section{INTRODUCTION}

Due to the aging of the population and rising rates of Alzheimer disease (AD), increasing efforts are made to develop tests allowing the early identification of aging individuals at risk of cognitive decline and dementia. Tests assessing recognition memory are often used in clinical settings to detect early cognitive manifestations of $A D$ in the aging population. It has previously been demonstrated that recognition performance is useful to predict individuals who will develop cognitive deficits over time (Peters, Villeneuve, \& Belleville, 2014; Rabin et al.,
2009). However, the sensitivity of these tests, and their accuracy in predicting impending dementia, would benefit from further refinement.

According to dual-process models, familiarity and recollection are two distinct and independent processes involved in the recognition of previously encountered material. Recollection is defined as a type of recognition that is accompanied with the retrieval of contextual details associated with the encoding episode. Familiarity, on the other hand, is perceived as recognition based on a sense, or a feeling of "knowing," that a stimulus has previously been encountered, despite the lack of retrieval of contextual details associated with the encoding episode. 
Recollection is viewed as a more controlled and elaborate form of cognitive process whereas familiarity is perceived as an automatic, rapid and implicit process. Recollection and familiarity are believed to work in a parallel and independent fashion to provide the global recognition experience. The dual-process theory of recognition is well established and has received a considerable amount of empirical support (see Yonelinas, 2002, for a review). However, a debate on the exact nature of recollection and familiarity and on the interplay between these two processes continues (Donaldson, 1996; Dunn, 2004; Hirshman \& Henzler, 1998; Inoue \& Bellezza, 1998; Smith, Wixted, \& Squire, 2011).

Studies investigating neurobiological substrates of recollection and familiarity suggest a functional dissociation of those two processes within the medial temporal lobe. More precisely, familiarity has been repeatedly associated with perirhinal/entorhinal areas whereas recollection has been associated with the hippocampus. This dissociation was highlighted in human lesion studies, which revealed selective deficits in recollection, with relative sparing of familiarity, following hippocampal lesion (Bowles et al., 2010; Vann et al., 2009). In contrast, selective deficits in familiarity, with sparing of recollection, were observed following lesions to the perirhinal area (Bowles et al., 2007, 2010; Bowles, O'Neil, Mirsattari, Poppenk, \& Köhler, 2011). This dissociation was further corroborated by functional magnetic resonance imaging (fMRI) studies. Indeed, results from multiple studies have shown an increased activation in hippocampal regions during the encoding of items later recognized on the basis of recollection and perirhinal activation during the encoding of items later recognized on the basis of familiarity (Daselaar, Fleck, \& Cabeza, 2006; Eldridge, Knowlton, Furmanski, Bookheimer, \& Engel, 2000; Ranganath et al., 2004). Consistently, a meta-analysis indicated that, across multiples fMRI studies, recollection was associated with hippocampal and parahippocampal activation during encoding or recognition (Diana, Yonelinas, \& Ranganath, 2007). On the other hand, perirhinal activation frequently predicted familiarity, but rarely recollection performance. Interestingly, regions that have been associated with recollection and familiarity are among the first to be targeted by neurofibrillary tangles, one of AD's neuropathological hallmarks (Braak \& Braak, 1991). Moreover, the presence of neurofibrillary tangles has been positively correlated with the severity of cognitive deficits in AD patients (Arriagada, Growdon, Hedley-Whyte, \& Hyman, 1992; Braak \& Braak, 1991, 1999). Taken together, these previous findings suggest that the assessment of familiarity and recollection in the aging population might be of great interest for the development of novel neuropsychological markers for AD.

The results of a recent meta-analysis looking at the effects of normal and neuropathological aging on recollection and familiarity point towards a decline in recollection with a preservation in familiaritybased recognition in cognitively normal aging individuals, as compared with young adults (Koen \& Yonelinas, 2014). The hippocampus is sensitive to the effects of aging and multiple studies have demonstrated structural and functional impairment in the hippocampus of normally aging individuals (Driscoll et al., 2003; Raz et al., 2005; Scahill et al., 2003). The impairment in recollection observed in normal aging individ- uals is therefore likely to be associated with reduction in structural and functional integrity of the hippocampus occurring naturally in the course of aging. Yet, very few studies have looked at relationships between hippocampus volumes and recollection performance in aging individuals. In a sample of cognitively normal aging individuals, Yonelinas and colleagues (2007) highlighted a negative relationship between age and hippocampal volume, which was in turn associated with recollection performance. In the same study, they further provided evidence for associations between entorhinal cortex volume and familiarity. However, they did not look at correlations with perirhinal cortex, an adjacent region that has frequently been linked with familiarity. A more recent study by Wolk, Dunfee, Dickerson, Aizenstein, and DeKosky (2011) also supports a double-dissociation between familiarity and recollection within the medial temporal lobe and showed that the left hippocampal volume significantly predicted recollection performance in a sample comprising cognitively normal aging individuals and individuals diagnosed with mild cognitive impairment $(\mathrm{MCl})$ or $A D$ (Wolk et al., 2011). On the other hand, the combined volume of extrahippocampal medial temporal lobe structures, such as the entorhinal, perirhinal and parahippocampal areas, was a stronger predictor of familiarity performance.

Interestingly, despite well-documented impairments in hippocampal functioning and reductions in recollection-based recognition, many studies have outlined a preservation of the overall recognition performance in cognitively normal aging individuals, as compared with younger adults (Craik \& McDowd, 1987; Fabiani \& Friedman, 1997; Schonfield \& Robertson, 1966). To explain this finding, we have previously hypothesized that aging individuals rely on familiarity at a greater extent than young individuals to sustain their recognition performance, despite a reduced recollection (Schoemaker, Gauthier, \& Pruessner, 2014). We further suggested that the increased rate of false alarms, or false recognition, frequently observed in aging individuals might be related to this increased reliance on familiarity. To define whether recollection and familiarity could be useful to differentiate normal from pathological aging, studies are needed characterize associations between recollection, familiarity and false alarms, as to obtain a better understanding of the recognition performance of cognitively normal aging individuals. The relationship between the dual-processes involved in recognition and structural integrity of medial temporal lobe regions also needs to be investigated more thoroughly.

In accordance with these needs, the objective of this article is to investigate three main hypotheses related to familiarity and recollection performance in aging individuals. The first hypothesis is that aging individuals rely on familiarity to a greater extent than young adults to support their recognition. Second, we hypothesize that the increased rate of false alarms frequently described in aging individuals is related to an increased reliance on familiarity. These two first hypotheses will be tested by comparing the performance of older and younger adults on recognition paradigm. The final hypothesis addressed in this article is that the decrease in recollection observed over the course of aging is associated with a decrease in hippocampal 
structural integrity, but independent of entorhinal and perirhinal cortices integrity. Accordingly, this last hypothesis will be investigated by means of correlations with structural neuroimaging data acquired in the older adults' cohort.

\section{MATERIALS AND METHODS}

\subsection{Participants}

Sixty young adults (age range: $18-25$ years) were recruited via the research participant pool of the Psychology Department at McGill University and obtained $1 \%$ extra credit for an eligible course within the Department as a result of their participation. Older adults were recruited via advertisements in community newspapers targeting the aging population to a fMRI study. To be included in this particular study, older adults had to meet the following criteria: aged between 60 and 80 years, MMSE score higher than 26 , absence of current or past neurological, psychiatric or severe medical conditions, no contra-indication for MRI, no current or past history of substance abuse, no current medication known to cross the blood brain barrier or alter cognitive functioning, more than 12 years of formal education, and a score in the normal range on the Geriatric Depression Scale (Yesavage et al., 1983) and Beck Anxiety Inventory (Beck et al., 1988). A total of 68 older adults were included in the study. Four aging individuals were excluded due to suspected cognitive impairment, as indicated by their performance on standardized cognitive tests. Four young adults and five older adults were further excluded from the analysis because of performance suggesting a lack of comprehension of task instructions (as indicated by an absence of variance in responses or a hit rate lower than 0.50 ), leaving 56 young adults (mean age: 20.38, SD 1.56) and 59 older adults (mean age: 66.97, SD $4.78)$ in the final cognitive analyses.

\subsection{Procedures}

The protocol of this study was reviewed and approved by the Douglas Mental Health University Institute's research ethics board. Young adults' subjects were tested in a single session, in which they were asked to complete a computerized task aiming to quantify recollection and familiarity. Older adults were tested in two separate sessions, the first session consisting of a cognitive evaluation, starting with the com-

TABLE 1 Summary of demographic and cognitive characteristics of older adults participants

\begin{tabular}{lll} 
& $M$ & SD \\
\hline Age & 66.97 & 4.78 \\
\hline M/F ratio & 1.52 & N/A \\
\hline Education & 15.12 & 2.54 \\
MMSE & 29.32 & 0.80 \\
\hline MoCA & 27.23 & 2.16 \\
\hline
\end{tabular}

Abbreviations: $M=$ mean; $S D=$ standard deviation; $M / F=$ male/female; $\mathrm{MMSE}=$ mini-mental state examination; $\mathrm{MoCA}=$ Montreal cognitive assessment. pletion of the computerized task to assess familiarity and recollection, and the second session consisting of an MRI scan performed at the Douglas Institute Brain Imaging Center. These two sessions were completed within a time interval of 4 weeks. All subjects were presented with information pertaining to the research project and provided written informed consent before the beginning of the study procedure.

\section{3 | Evaluation of basic cognitive functions}

Older adults underwent a cognitive test battery to allow exclusion of individuals with cognitive impairments, as defined with a performance lower than 1.5 standard deviations from the age-adjusted mean. The tests administered included: (1) Mini-Mental Status Exam (Folstein, Folstein, \& McHugh, 1975); (2) Montreal Cognitive Assessment (Nasreddine et al., 2005); (3) Rey Auditory Verbal Learning Test (Schmidt, 1996); (4) letter (FAS) and category fluency (animals) (Tombaugh, Kozak, \& Rees, 1999); (5) Trail Making Test A and B (Reitan, 1992); (6) Symbol-Digit Modality task (Smith, 1973); (7) Boston Naming Test (Kaplan, Goodglass, \& Weintraub, 1983); (8) Stroop Colour-Word interference Test (Golden \& Freshwater, 2002); (9) Digit Span subtest of the Wechsler Adult Intelligence Scale III (Wechsler, 1997) and (10) Matrix reasoning subtest from the Wechsler Abbreviated Scale of Intelligence II (Wechsler \& Hsiao-Pin, 2011). Demographic and global cognitive characteristics of older participants are summarized in Table 1.

\subsection{Structural neuroimaging analysis}

\subsubsection{MR imaging}

MR scans were acquired in older adults only. Scans were obtained on a 3Tesla Siemens Magnetom TrioTim scanner (Siemens Healthineers, Erlangen, Germany), using a 32-channel head coil. A T1-weighted MRI scan was collected for each participant using a sagittal volumetric magnetization prepared rapid gradient echo (3D MP-RAGE) and the following acquisition parameters: echo time $(\mathrm{TE})=2.98 \mathrm{~ms}$, repetition time $(T R)=2,300$ $\mathrm{ms}$, flip angle of $9^{\circ}$, field of view $=256 \mathrm{~mm}$, acquisition matrix $=256 \times$ $256 \times 176$, voxel dimensions $=1.0 \times 1.0 \times 1.2$. Out of the 59 aging individuals included in recollection/familiarity analyses, 3 were unable to complete the MRI examination due to anxiety or discomfort. Consequently, only 56 individuals were included in volumetric-based analyses.

\subsubsection{Image pre-processing}

Acquired T1 images were denoized (Coupé et al., 2008, ) and corrected for non-uniformity (Sled, Zijdenbos, \& Evans, 1998). Images were subsequently linearly registered to MNI standard space (Collins, Neelin, Peters, \& Evans, 1994). A visual quality control was performed to ensure quality of the image preprocessing. None of the scan had to be excluded due to poor preprocessing.

\subsubsection{Hippocampal, entorhinal and perirhinal volume estimation}

Pre-processed MPRAGE images were automatically segmented using a previously described technique (Coupé et al., , 2011). For each participant, automatically segmented hippocampal, entorhinal and perirhinal 
volumes were extracted and manually corrected by an expert in neuroanatomy, using previously published segmentation guidelines (Pruessner et al., 2000, 2002). Intra-rater reliability of the manual correction was assessed on the basis of 18 repeated segmentations. The reliability was satisfactory, as indicated by intraclass correlation coefficients of 0.93 for hippocampus (HP) volumes, 0.91 for perirhinal cortex (PC) volumes and 0.96 for entorhinal $(E C)$ volumes. Finally, all volumes were corrected for total intracranial volume, an approach previously recommended to normalize volumes across different subjects and reduce biases associated with brain size (Free et al., 1995; Whitwell, Crum, Watt, \& Fox, 2001). Results are reported for the right hemisphere $(\mathrm{rHP} / \mathrm{rPC} / \mathrm{rEC})$ volumes, the left hemisphere (IHP/IPC/IEC) volumes and the total volumes (left + right hemisphere volumes; tHP/tPC/tEC).

\subsection{Quantification of familiarity and recollection}

\subsubsection{Procedure}

Subjects were placed in front of a laptop computer (screen size: 17 inches, resolution: $1,280 \times 800$ pixels) and presented with the computerized task programmed using SuperCard ${ }^{\circledR}$ software Version 4.72 (Solutions Etcetera, 2012, California, USA). To reduce situational variability, task instructions were written on the screen. However, participants were encouraged to refer to the experimenter if they needed any further instructions or clarifications. The task took between 20 and 30 min to complete.

\subsubsection{Stimuli}

Sixty-nine black and white neutral Caucasian faces were selected from the FACES database (Ebner, Riediger, \& Lindenberger, 2010). Of the selected faces, 23 were used as targets during the first encoding condition, 23 were used as targets during the second encoding condition and the remaining 23 faces were used as distractors during recognition. Targets and distractors' lists were equated for gender and age of presented faces.

\subsubsection{Experimental paradigm}

The experimental paradigm used in this study consists in a variation the process dissociation procedure developed by Jacoby (1991). In contrast with the standard process dissociation procedure, the current paradigm allows the estimation of recollection and familiarity estimates from a unique recognition phase. This type of variation was done in previous studies aiming to quantify the contribution of recollection and familiarity and using a dissociation framework (Smith \& Knight, 2002; Wilding \& Rugg, 1997). A unique recognition trial has the advantage of being simpler to understand, faster to administer and allows to avoid certain methodological biases associated with different response biases that may arise from different conditions.

In the encoding phase, participants were presented with a total of 46 faces in two separate conditions ( 23 faces per condition). Within each encoding condition, faces were presented one at a time, in random order, for $4 \mathrm{~s}$ and were separated by a $100 \mathrm{~ms}$ inter-stimulus interval. In the first encoding condition, named the "blue condition," faces were presented on the right side of the screen on a blue background. For each face, participants were asked to answer the question
"Does this person look upset?" by clicking on either the "yes" or "no" button on the screen. In the second encoding condition, named the "red condition," faces were presented on the left side of the screen on a red background. This time, for each face, participants were prompted to answer the question "Does this person look friendly?," again by clicking on either the "yes" or "no" button on the screen. These questions were meant to favor a deeper encoding and to provide an additional anchor for subsequent recognition. Participants were informed that there was no right and wrong answer to these questions and encouraged to answer on the basis of their subjective feelings. The order of presentation of the two encoding conditions was counterbalanced across subjects, that is, half of participants were presented with the blue condition first and the other half of participants were presented with the red encoding condition first.

The recognition task immediately followed the encoding procedure. Participants were presented with the 46 faces studied during the encoding phase as well as 23 new unseen faces (distractors). Each face was presented one at a time in the center of the screen. The recognition was then performed in a two-step procedure. First, participants were asked to judge whether the presented face was shown during the encoding phase, regardless of the encoding condition, and to respond by clicking on either the "new" or "old" button on the screen. Second, if participants identified a face as being "old," they were asked to determine whether it was shown in the "blue" or the "red" encoding condition. At this point, participants were reminded of contextual differences between the two encoding conditions. To avoid biases associated with guesses, participants were also given the option to answer "don't know." Therefore, participants had to respond to the contextual question by clicking on either the "red condition," the "blue condition" or the "don't know" button on the screen. The recognition was self-paced. Participants were given three practice trials (2 target faces and 1 distractor, same for all participants) before starting the recognition task. Participants were encouraged to ask any questions if the procedure seemed unclear. Following the completion of practice trials, participants were presented with the remaining 44 targets and 22 distractor faces, in a randomized presentation order.

\subsubsection{Quantification of responses and statistical analysis}

Results from the computerized task were quantified as follow. The hit rate $(H)$ was defined as the proportion of faces correctly recognized as being old. The false alarm rate $(F A)$ was defined as the proportion of new faces incorrectly categorized as being old. Recollection and familiarity scores were derived according to the framework proposed by Jacoby (1991). In this experiment, the inclusion score (I) was defined as the proportion of old faces correctly identified as being old. The exclusion score $(E)$ was computed as the proportion of hits associated with either an incorrect source attribution or a "don't know" response. As described by Jacoby (1991), recognition responses contributing to the inclusion score, I, can be supported by recollection, $R$, and/or familiarity, $F$, (i.e., $I=R+F(1-R)$ ). On the other hand, the exclusion score, $E$, implies involvement of familiarity in the absence of recollection $(E=F$ $(1-R))$. Thus, solving these equations allows the isolation of $R$ and $F$ variables: $R=(I-E)$ and $F=(E /(1-R))$. Finally, to quantify the relative 
contribution of recollection to the overall recognition at the individual level, a "recollection ratio" (RR) was computed using the proportion of hits with correct source attribution $(R H)$ relative to the overall hit rate (Recollection ratio $=R H / H$ ). Statistical analyses were carried out using IBM SPSS software version 20. An multivariate analysis of variance (MANOVA) was used to compare the cognitive performance of young subjects and older adults, using the group as the independent variable (young vs older adults) and indexes of recognition performance as dependent variables (hit rate, false alarm rate, recollection, familiarity and recollection ratio). In older adults only, simple Pearson correlations analyses were performed to investigate relationships between cognitive performance on the recollection/familiarity task and medial temporal lobe volumes. Finally, multiple linear regression analyses were conducted to define whether each medial temporal lobe volume was a significant predictor of recollection or familiarity performance, together with the covariates of age, gender and education.

\section{RESULTS}

\subsection{Age-related differences in recollection and familiarity: associations with false alarms}

Results of the MANOVA contrasting the recognition performance of young and older participants are presented in Table 2. The hit rate did

TABLE 2 Summary of group performance on the recollection/familiarity task

\begin{tabular}{|c|c|c|c|c|c|}
\hline & \multicolumn{2}{|c|}{ Young adults } & \multicolumn{2}{|c|}{ Older adults } & \multirow[b]{2}{*}{$F(1,113)$} \\
\hline & $M$ & $S D$ & $M$ & $S D$ & \\
\hline Hit rate/inclusion score & 0.70 & 0.09 & 0.72 & 0.12 & 0.39 \\
\hline Exclusion score & 0.36 & 0.09 & 0.44 & 0.13 & $12.23^{* *}$ \\
\hline$P(“ D K ”$ response) & 0.14 & 0.12 & 0.18 & 0.18 & 1.88 \\
\hline $\begin{array}{l}P \text { (Incorrect source } \\
\text { attribution) }\end{array}$ & 0.23 & 0.09 & 0.26 & 0.10 & 3.51 \\
\hline False alarm rate & 0.11 & 0.09 & 0.21 & 0.16 & $16.70^{* * *}$ \\
\hline Recollection estimate & 0.34 & 0.10 & 0.27 & 0.11 & $9.19^{* *}$ \\
\hline Familiarity estimate & 0.55 & 0.12 & 0.61 & 0.16 & $4.27^{*}$ \\
\hline Recollection ratio & 0.48 & 0.13 & 0.38 & 0.14 & $12.51^{* * *}$ \\
\hline
\end{tabular}

Note. $M=$ mean, $S D=$ standard deviation, Hit rate/Inclusion score-proportion of correct recognition responses on the yes/no recognition task (P Old/Old), Exclusion score-proportion of correct recognition responses on the yes/no recognition task that are accompanied with a failure to provide correct source attribution, divided into: $\mathrm{P}$ ("DK" response)-proportion of "Don't know" responses contributing to the exclusion score, $\mathrm{P}$ (Incorrect source attribution)-proportion of recognition responses with incorrect source attribution contributing to the exclusion score, False alarm rate-proportion of new items incorrectly endorsed as being "old" (P Old/New), Recollection and Familiarity estimates-estimates derived from Jacoby (1991) process dissociation framework formulas, Recollection ratio-proportion of correct recognition responses with correct source attribution relative to the total number of correct recognition responses (P Correct source attribution/Hit), F-Fisher test indicating the magnitude of differences between young and old adults.

${ }^{*} p<.05,{ }^{* *} p<.01,{ }^{* * *} p<.001$ not differ between young and older adults $(F=0.39, p>.05)$. However, there was a significant difference in the rate of false alarms between groups $(F=16.70, p<.001)$. Young adults showed an increased recollection estimate $(F=9.19 p<.01)$, and reduced familiarity estimate $(F=4.27, p<.05)$, as compared with older adults. As indicated by a significantly superior recollection ratio $(F=12.51, p=.001)$, young adults relied on recollection to a greater extent than older adults to support their overall recognition performance. When assessing relationships between recollection, familiarity and false alarms, Pearson correlations indicated a significant positive association between familiarity estimates and false alarm rates $(r=0.30, p=.001)$. Recollection was not significantly associated with the rate of false alarms $(r=-0.08$, $p>.05)$. When considering groups separately, correlations between familiarity and false alarms remained significant in the older adult group $(r=0.34, p<.01)$, but not in young adults $(r=0.06, p>.05)$. Further, in the older adult group, the correlation between familiarity and false alarms remained significant after controlling for age in a partial correlation $(r=0.36, p<.01)$.

\subsection{Recollection and familiarity in aging individuals: relationships with medial temporal lobe structures}

\subsubsection{Associations between normalized regional volumes and age}

Pearson correlations were computed to assess the associations between normalized hippocampal, entorhinal, and perirhinal volumes and age. Age was significantly and negatively associated with normalized hippocampal volumes (IHP: $r=-0.47, r H P$ : $r=-0.38$, tHP: $r=-0.43 ; p<.01$ ). Marginal correlations were observed between age and the normalized volume of the left (IEC: $r=-0.25, p=.06$ ), right (rEC: $r=-0.19, p>.05$ ) and total (tEC: $r=-0.24 ; p=.07$ ) entorhinal cortex. Marginal correlations were also found between age and the normalized volume of the left (IPC: $r=-0.26, p=.05$ ), right ( $r P C$ : $r=-0.21, p>.05)$ and total (tPC: $r=-0.25 ; p=.06)$ perirhinal cortex.

\subsubsection{Associations with normalized hippocampal volumes}

Pearson correlations were computed to assess the associations between performance on the recognition task and hippocampus integrity, as estimated by normalized hippocampal volumes. A trend for significance was found between the hit rate and the IHP $(r=0.26$, $p<.10)$, rHP $(r=0.23, p<.10)$ and tHP $(r=0.24, p<.10)$ normalized hippocampus volumes. The recollection estimate was positively and significantly associated with the normalized volumes of the IHP $(r=0.51, p<0.001)$, the $\mathrm{rHP}(r=0.50, p<0.001)$ and the tHP $(r=0.51, p<0.001)$. There was no significant correlation between familiarity estimate and the right, left or total normalized hippocampal volume (IHP: $r=0.07$, rHP: $r=0.04$, tHP: $r=0.06 ; p>.05$ ). Similarly, no significant correlation between hippocampal volume and false alarm rates were found (IHP: $r=-0.05$, rHP: $r=-0.01$, tHP: $r=-0.03$; $p>$.05). A multiple linear regression analysis was computed to predict recollection performance based on age, education, gender and total hippocampal volumes of older adult participants. The total hippocampal volume was found to be the only significant predictor of recollection 
performance $(\beta=0.51, \quad p<.001)$, while partial effects of age $(\beta=-0.001, \quad p>.05)$, gender $(\beta=0.04, \quad p>.05)$ and education $(\beta=0.01, p>.05)$ did not significantly contribute to the model and were excluded. A model including the total hippocampal volume as the single predictor accounted for $25 \%$ of the variance (adjusted value) in recollection $(F(1,54)=19.11, p<.001)$. In a different regression model to predict familiarity performance on the basis on age, education, gender and total hippocampal volume of older adult participants, none of the covariables were found to significantly predict familiarity and the model was not significant $(F(4,51)=0.44, p>.05)$.

\subsubsection{Associations with normalized entorhinal cortex volumes}

No significant association was found between normalized entorhinal cortex volumes and measures of recognition performance. More precisely, the hit rate was not significantly associated with entorhinal volumes (rEC: $r=0.04$, IEC: $r=0.05$, tEC: $r=0.05 ; p>.05$ ). Similarly, no significant association was found with recollection estimates ( $r E C$ $r=0.26$, IEC $r=0.17$, tEC $r=0.25 ; p>.05$ ), familiarity estimates ( $r E C$ : $r=-0.04$, IEC: $r=-0.00$, tEC: $r=0.02 ; p>.05)$ or false alarm rates ( $r E C: r=0.04$, IEC: $r=-0.18$, tEC: $r=-0.09 ; p>.05$ ). When computing multiple linear regression analyses to predict recollection or familiarity performance based on age, education, gender and total entorhinal cortex volume, none of the variables were identified as a significant predictor of performance and both regression models were not statistically significant (recollection: $(F(4,51)=1.19, p>.05$; familiarity: $F(4,51)=0.39, p>.05)$.

\subsubsection{Associations with normalized perirhinal cortex volumes}

When looking at correlations with normalized perirhinal cortex volumes, no significant association was found with the overall hit rate ( $r P C: r=0.02$, IPC: $r=0.14$, tPC: $r=0.09 ; p>.05$ ). Similarly, no significant association was found with either recollection ( $r P C: r=0.13$, IPC: $r=0.10$, tPC: $r=0.12 ; p>.05$ ) or familiarity estimates ( $r P C: r=0.00$, IPC: $r=0.15$, tPC: $r=0.08 ; p>.05$ ). Correlations between perirhinal volumes and false alarm rates also failed to reach statistical significance (rPC: $r=-0.00$, IPC: $r=-0.10$, tPC: $r=-0.06$ ). When computing multiple linear regression analyses to predict recollection or familiarity performance based on age, education, gender and total perirhinal cortex volume, none of the variables were identified as a significant predictor of performance and both regression models were not statistically significant (recollection: $F(4,51)=0.58, p>.05$; familiarity: $F(4,51)=$ $0.49, p>.05)$.

\section{DISCUSSION}

In this article, we compared the recollection and familiarity performance of young and older adults. We also made associations with the rate of false alarms. In older adults, we further investigated relationships between these recognition variables and structural integrity of the hippocampus as well as the entorhinal and perirhinal cortices. Overall, we observed a decrease in recollection and increase in familiarity-based recognition in older adults, as compared with young adults. In older adults, familiarity, but not recollection, was significantly and positively associated with the rate of false alarms. Significant positive correlations were found between recollection and hippocampal volumes, suggesting that the decrease in recollection observed in older adults is likely associated with a reduction in hippocampal structural integrity. On the other hand, no relationship was found between recognition performance and volumes of perirhinal or entorhinal cortices.

One of our initial hypotheses was that older adults rely on familiarity at a greater extent than young adults to support their recognition. Our results demonstrate that older and younger participants showed an equivalent number of correct recognition (i.e. hits). However, as compared with young adults, older adults showed a decrease in recollection together with an increase reliance on familiarity. In accordance with the present findings, results from a recent meta-analysis highlighted a recollection decrease in aging individuals (Koen \& Yonelinas, 2014). An increase in familiarity in aging individuals has also previously been reported in the literature. For example, Angel and colleagues (2013) found that familiarity responses were more common in older participants than in young participants, especially when the task was simple (Angel et al., 2013). Using a facial recognition paradigm, Bastin and Van der Linden investigated the frequency of "remember" and "know" responses in young and aging individuals (Bastin \& Van der Linden, 2003). They demonstrated that, while recollection responses were less frequent in older adults, the rate of familiarity responses was increased. The authors further noted that the higher frequency of familiarity responses in aging individuals was more evident in forcedchoice paradigms as opposed to yes-no recognition tasks. Nonetheless, there seems to be an important heterogeneity in results of studies investigating familiarity performance in aging individuals (Koen \& Yonelinas, 2014). Methodological variability appears to account for some of the disparity in results across studies. Consistently, it has already been demonstrated that methodological aspects, such as the experimental paradigm (Koen \& Yonelinas, 2014) and stimulus-type (Belleville, Ménard, \& Lepage, 2011; Embree, Budson, \& Ally, 2012), influence performance in recollection and familiarity. It is also possible that other factors, such as group differences in the level of risk factors for $A D$, contributed to the observed variability in findings. Future studies are therefore needed to better understand factors behind the important discrepancy in results of studies investigating familiarity performance in aging individuals.

A large body of evidence indicates that the recognition performance of aging individuals is characterized by an increased tendency for false alarms (see McCabe, Roediger, McDaniel, \& Balota, 2009; Schacter, Koutstaal, \& Norman, 1997 for reviews). Accordingly, our results support an increased rate of false alarms in older adults, as compared with young adults. In this article we aimed to define whether the increased rate of false alarms often described in the aging population was associated with an increased reliance on familiarity-based recognition. In older adults, our results indicate a significant positive correlation between familiarity and false alarms. On the other hand, the false alarm rate was not associated with recollection. Thus, false alarms 
seem to be related to an increased reliance on familiarity rather than a decrease in recollection-based recognition. In the older adult group, the positive correlation between false alarms and familiarity remained significant after controlling for age. Interestingly, the correlation between familiarity and false alarms was not significant in young adults. Previous evidence suggests that the recognition performance of younger adults is less affected by the perceptual similarity of the encoding material (Schacter, Israel, \& Racine, 1999). Indeed, Schacter et al. (1999) showed that aging individuals are more prone to false alarms when confronted with perceptually similar stimuli. They proposed that this was possibly due to the fact that aging individuals tend to rely on a global and general encoding strategy, that may be less effective when confronted with stimuli of high perceptual similarity. As faces are highly similar at the perceptual level, this type of stimuli likely provides little distinctiveness. Thus, it is possible that, in the present experiment, aging individuals are selectively disadvantaged because of the low distinctiveness between stimuli, consequently leading to a greater rate of false alarms and increased reliance on familiarity-based recognition. In turn, this could also explain the lack of correlation between false alarms and familiarity in young adults. However, to gain a better understanding of this discrepancy, future studies should aim to replicate these findings using material that allows better differentiation or distinctiveness across stimuli.

The secondary aim of this study was to investigate relationships between recognition performance and structural integrity of the hippocampus, entorhinal and perirhinal areas in older adults. Overall, our results support positive associations between recollection and normalized hippocampus volumes. In a regression model including age, education, gender and hippocampal volume as variables, hippocampal volume was found to be the only significant predictor, accounting itself for $25 \%$ of the variance in recollection performance. These results are consistent with the few previous articles that have investigated relationships between volumetric measures of medial temporal lobe regions and the dual-processes of recognition and support an association between recollection and hippocampal structural integrity (Wolk et al., 2011; Yonelinas et al., 2007). The relationship between hippocampus and recollection has also frequently been highlighted in functional neuroimaging studies (Daselaar, Fleck, Dobbins, Madden, \& Cabeza, 2006; Diana et al., 2007; Eldridge et al., 2000). Due to the nature of our experimental task, our findings can also be paralleled with the multiple reports demonstrating a link between the hippocampus and associative or contextual memory (Daselaar, Fleck, Dobbins, et al., 2006; Davachi, 2006; Sullivan Giovanello, Schnyer, \& Verfaellie, 2004; Yonelinas, Hopfinger, Buonocore, Kroll, \& Baynes, 2001). Hippocampus volumes did not significantly correlate with the hit rate, the familiarity estimate nor the false alarm rate. Thus, recollection might provide a more precise cognitive marker of hippocampal integrity than these other measures of recognition performances.

No significant association was found between any recognition measures and two other studied medial temporal lobes regions, namely the entorhinal and perirhinal cortices. This diverges from previous reports supporting associations between structural integrity of these regions and familiarity-based recognition. For example, Yonelinas and colleagues demonstrated that, while the entorhinal region correlated more strongly with recognition performance, the recall performance was associated with the hippocampus (Yonelinas et al., 2007). These findings were interpreted as evidence for a functional double dissociation between the neuroanatomical substrates of recollection and familiarity. However, these findings are difficult to contrast with the results of the present experiment as they rely on a very different experimental method to estimate recollection and familiarity performances. More precisely, recollection and familiarity are assessed contrasting the recall and recognition performance, a method associated with several limitations and likely to provide an imprecise portrait of recollection and familiarity (Yonelinas, 2002). In a different article, Wolk et al. investigated associations between recognition performance and medial temporal lobe structural integrity in non-demented older individuals as well as in patients with $\mathrm{MCl}$ or early AD (Wolk et al., 2011). The results of their study highlighted a significant decline in both recollection and familiarity in patients with $\mathrm{MCl}$ or $\mathrm{AD}$ in contrast to control subjects. They further showed that the combined volumes of the entorhinal, perirhinal and parahippocampal cortices significantly predicted familiarity, but did not predict recollection performance. The authors did not provide the predictive value of each extrahippocampal region separately. A major distinction of the article from Wolk and colleagues, as compared with this study, consists in the inclusion of patients with objective cognitive impairments and AD. While hippocampal integrity is sensitive to processes associated with normal and pathological aging, some evidence suggests that the entorhinal and perirhinal cortices are relatively spared during normal aging and specifically altered in the course of AD (Raz, Rodrigue, Head, Kennedy, \& Acker, 2004). Accordingly, while the results of this study support significant and strong negative correlations between normalized hippocampal volumes and age, perirhinal and entorhinal volumes were less strongly associated with age, with correlations of marginal significance. Familiarity-based recognition has been previously linked to the integrity of the rhinal region (Bowles et al., 2007, 2010), thus it can be expected to find familiarity impairments in individuals with alterations in this brain area, such as individuals with AD. The sample used in this study however only included aging individuals with no overt cognitive impairment. It is therefore possible that rhinal alterations in these individuals were not pronounced enough to show associations with familiarity performance. Thus, while the present results do not support associations between entorhinal/perirhinal cortices volume and familiarity, it also does not exclude this possibility. Consequently, more studies are needed in patients with documented brain lesions to clarify relationships between familiarity and structural integrity of rhinal region. Another possible reason behind the lack of correlation between rhinal volumes and recognition performance consists in the anatomical variability of these structures. The collateral sulcus, that importantly determines the anatomical characteristics of the rhinal areas, is highly variable across individuals (Pruessner et al., 2002). Consequently, the shape of the entorhinal and perirhinal cortices is more variable than that of the hippocampus. The presence of bifurcations and/or interruptions of the collateral sulcus will inevitably increase or decrease the volume of the entorhinal and perirhinal cortices, without necessarily 
reflecting pathological processes. In the context of cross-sectional designs, the relevance of volumetric data in the investigation of entorhinal and perirhinal atrophy is therefore possibly limited by the important variability in shape associated with this area. Thus, other measures of structural integrity, such as the cortical thickness, could potentially provide an interesting alternative to reduce the impact of perirhinal and entorhinal shape variability. The investigation of associations between recognition processes and other cortical regions could also be of great relevance. Some models have previously suggested that age-related cognitive impairments could be linked with a deterioration of the frontal lobes and related cognition functions (Nyberg et al., 2010; West, 1996). Previous studies have revealed impairments in recollection-based processes, including source monitoring and temporal ordering, in individuals with prefrontal cortex lesions (Duarte, Ranganath, \& Knight, 2005; Janowsky, Shimamura, \& Squire, 1989; Johnson, O'Connor, \& Cantor, 1997; Shimamura, Janowsky, \& Squire, 1990; Simons et al., 2002). Consistently, in animals, lesions to the medial prefrontal cortex were found to selectively disrupted recollection (Farovik, Dupont, Arce, \& Eichenbaum, 2008). However, these findings are not equivocal in the literature and two studies suggest a preservation of recollection-based recognition in patients with frontal lobe lesions (Aly, Yonelinas, Kishiyama, \& Knight, 2011; MacPherson et al., 2008). Consequently, future studies should aim to better characterize the influence of frontal lobes functional and structural integrity with regards to the age-related decline in recollection.

A few limitations of this study merit consideration. First, the neuroimaging data were only available in the older adults group and not for younger subjects. Thus, the difference in performance of these two groups on the recollection/familiarity task could not be linked with structural differences in brain anatomy. Second, it is possible that the observed positive association between familiarity and false alarms is mediated by variations in recognition confidence. Indeed, a lower confidence in recognition responses might lead to an increased reliance on familiarity and/or an increase rate of false alarms. The inclusion of a rating of confidence would have been informative and would have allow to depict a more precise portrait of the interplay between these different recognition variables. Finally, this study has been designed to focus on dual-process theories rather than other conceptual models of recognition. Thus, although the results of this study were interpreted within a dual-process framework, we acknowledge that they could be interpreted on the basis of different theoretical perspectives.

To conclude, the present results indicate that older adults tend to rely on familiarity at a greater extent than young adults to support their recognition judgment. The increase use of familiarity-based recognition in older adults is positively associated with an increase in false alarms. On the other hand, recollection was not associated with the rate of false alarms. When investigating associations between medial temporal lobe structures and recognition performance in older adults, the results supported a positive association between recollection and normalized hippocampal volumes. No association between recognition measures and perirhinal or entorhinal cortices were observed, perhaps due to the anatomical variability of these regions. At this point, the results of this study suggest that recollection is a more precise marker of hippocam- pal integrity than other typical measures of recognition performance, such as the hit rate or the false alarm rate. Consequently, recollection performance could provide a superior indication of aging individuals at risk of developing $A D$.

\section{REFERENCES}

Aly, M., Yonelinas, A. P., Kishiyama, M. M., \& Knight, R. T. (2011). Damage to the lateral prefrontal cortex impairs familiarity but not recollection. Behavioural Brain Research, 225(1), 297-304.

Angel, L., Bastin, C., Genon, S., Balteau, E., Phillips, C., Luxen, A., ... Collette, F. (2013). Differential effects of aging on the neural correlates of recollection and familiarity. Cortex, 49(6), 1585-1597.

Arriagada, P. V., Growdon, J. H., Hedley-Whyte, E. T., \& Hyman, B. T. (1992). Neurofibrillary tangles but not senile plaques parallel duration and severity of Alzheimer's disease. Neurology, 42(3), 631-639.

Bastin, C., \& Van der Linden, M. (2003). The contribution of recollection and familiarity to recognition memory: A study of the effects of test format and aging. Neuropsychology, 17(1), 14-24.

Beck, Aaron T, Epstein, Norman, Brown, Gary, \& Steer, Robert A. (1988). An inventory for measuring clinical anxiety: psychometric properties. Journal of consulting and clinical psychology, 56(6), 893.

Belleville, S., Ménard, M.-C., \& Lepage, É. (2011). Impact of novelty and type of material on recognition in healthy older adults and persons with mild cognitive impairment. Neuropsychologia, 49(10), 2856-2865.

Bowles, B., Crupi, C., Mirsattari, S. M., Pigott, S. E., Parrent, A. G., Pruessner, J. C., ... Köhler, S. (2007). Impaired familiarity with preserved recollection after anterior temporal-lobe resection that spares the hippocampus. Proceedings of the National Academy of Sciences, 104(41), 16382-16387.

Bowles, B., Crupi, C., Pigott, S., Parrent, A., Wiebe, S., Janzen, L., \& Köhler, S. (2010). Double dissociation of selective recollection and familiarity impairments following two different surgical treatments for temporal-lobe epilepsy. Neuropsychologia, 48(9), 2640-2647.

Bowles, B., O'neil, E. B., Mirsattari, S. M., Poppenk, J., \& Köhler, S. (2011). Preserved hippocampal novelty responses following anterior temporal-lobe resection that impairs familiarity but spares recollection. Hippocampus, 21(8), 847-854.

Braak, H., \& Braak, E. (1991). Neuropathological stageing of Alzheimerrelated changes. Acta Neuropathologica, 82(4), 239-259.

Braak, H., \& Braak, E. (1999). Cortical destruction and cell death in Alzheimer's disease. In V. E. Koliatsos \& R. R. Ratan (Eds.), Cell death and diseases of the nervous system. (pp. 497-510). Totowa, NJ: Humana Press.

Collins, D. L., Neelin, P., Peters, T. M., \& Evans, A. C. (1994). Automatic $3 \mathrm{D}$ intersubject registration of $\mathrm{MR}$ volumetric data in standardized Talairach space. Journal of Computer Assisted Tomography, 18(2), 192-205.

Coupé, P., Manjón, J. V., Fonov, V., Pruessner, J., Robles, M., \& Collins, D. L. Nonlocal Patch-Based Label Fusion for Hippocampus Segmentation. Medical Image Computing and Computer-Assisted Intervention, 13, 129-136.

Coupé, P., Manjón, J. V., Fonov, V., Pruessner, J., Robles, M., \& Collins, D. L. (2011). Patch-based segmentation using expert priors: Application to hippocampus and ventricle segmentation. Neurolmage, 54(2), 940-954.

Coupé, P., Yger, P., Prima, S., Hellier, P., Kervrann, C., \& Barillot, C. (2008). An optimized blockwise nonlocal means denoising filter for 3D magnetic resonance images. Medical Imaging, IEEE Transactions on, 27(4), 425-441. 
Craik, F. I., \& McDowd, J. M. (1987). Age differences in recall and recognition. Journal of Experimental Psychology: Learning, Memory, and Cognition, 13(3), 474-479.

Daselaar, S. M., Fleck, M. S., \& Cabeza, R. (2006). Triple dissociation in the medial temporal lobes: Recollection, familiarity, and novelty. Journal of Neurophysiology, 96(4), 1902-1911.

Daselaar, S. M., Fleck, M. S., Dobbins, I. G., Madden, D. J., \& Cabeza, R. (2006). Effects of healthy aging on hippocampal and rhinal memory functions: An event-related fMRI study. Cerebral Cortex, 16(12), 1771-1782.

Davachi, L. (2006). Item, context and relational episodic encoding in humans. Current Opinion in Neurobiology, 16(6), 693-700.

Diana, R. A., Yonelinas, A. P., \& Ranganath, C. (2007). Imaging recollection and familiarity in the medial temporal lobe: A three-component model. Trends in Cognitive Sciences, 11(9), 379-386.

Donaldson, W. (1996). The role of decision processes in remembering and knowing. Memory \& Cognition, 24(4), 523-533.

Driscoll, I., Hamilton, D. A., Petropoulos, H., Yeo, R. A., Brooks, W. M., Baumgartner, R. N., \& Sutherland, R. J. (2003). The aging hippocampus: Cognitive, biochemical and structural findings. Cerebral Cortex, 13(12), 1344-1351.

Duarte, A., Ranganath, C., \& Knight, R. T. (2005). Effects of unilateral prefrontal lesions on familiarity, recollection, and source memory. The Journal of Neuroscience, 25(36), 8333-8337.

Dunn, J. C. (2004). Remember-know: A matter of confidence. Psychological Review, 111(2), 524-542.

Ebner, N. C., Riediger, M., \& Lindenberger, U. (2010). FACES-A database of facial expressions in young, middle-aged, and older women and men: Development and validation. Behavior Research Methods, 42(1), 351-362.

Eldridge, L. L., Knowlton, B. J., Furmanski, C. S., Bookheimer, S. Y., \& Engel, S. A. (2000). Remembering episodes: A selective role for the hippocampus during retrieval. Nature Neuroscience, 3(11), 1149-1152.

Embree, L. M., Budson, A. E., \& Ally, B. A. (2012). Memorial familiarity remains intact for pictures but not for words in patients with amnestic mild cognitive impairment. Neuropsychologia, 50(9), 2333-2340.

Fabiani, M., \& Friedman, D. (1997). Dissociations between memory for temporal order and recognition memory in aging. Neuropsychologia, 35(2), 129-141.

Farovik, A., Dupont, L. M., Arce, M., \& Eichenbaum, H. (2008). Medial prefrontal cortex supports recollection, but not familiarity, in the rat. The Journal of Neuroscience, 28(50), 13428-13434.

Folstein, M. F., Folstein, S. E., \& McHugh, P. R. (1975). Mini-mental state": A practical method for grading the cognitive state of patients for the clinician. Journal of Psychiatric Research, 12(3), 189-198.

Free, S., Bergin, P., Fish, D., Cook, M., Shorvon, S., \& Stevens, J. (1995). Methods for normalization of hippocampal volumes measured with MR. American Journal of Neuroradiology, 16(4), 637-643.

Golden, C., \& Freshwater, S. (2002). A manual for the adult Stroop Color and Word Test (Stoelting Ed.). Chicago, IL: Stoelting.

Hirshman, E., \& Henzler, A. (1998). The role of decision processes in conscious recollection. Psychological Science, 9(1), 61-65.

Inoue, C., \& Bellezza, F. S. (1998). The detection model of recognition using know and remember judgments. Memory \& Cognition, 26(2), 299-308.

Jacoby, L. L. (1991). A process dissociation framework: Separating automatic from intentional uses of memory. Journal of Memory and Language, 30(5), 513-541.

Janowsky, J. S., Shimamura, A. P., \& Squire, L. R. (1989). Source memory impairment in patients with frontal lobe lesions. Neuropsychologia, 27 (8), 1043-1056.
Johnson, M. K., O'Connor, M., \& Cantor, J. (1997). Confabulation, memory deficits, and frontal dysfunction. Brain and cognition, 34(2), 189-206.

Kaplan, E., Goodglass, H., \& Weintraub, S. (1983). Boston naming test. Philadelphia, PA: Lea \& Febiger.

Koen, J. D., \& Yonelinas, A. P. (2014). The effects of healthy aging, amnestic mild cognitive impairment, and Alzheimer's disease on recollection and familiarity: A meta-analytic review. Neuropsychology Review, 24(3), 332-354.

MacPherson, S. E., Bozzali, M., Cipolotti, L., Dolan, R. J., Rees, J. H., \& Shallice, T. (2008). Effect of frontal lobe lesions on the recollection and familiarity components of recognition memory. Neuropsychologia, 46(13), 3124-3132.

McCabe, D. P., Roediger, H. L., McDaniel, M. A., \& Balota, D. A. (2009). Aging reduces veridical remembering but increases false remembering: Neuropsychological test correlates of remember-know judgments. Neuropsychologia, 47(11), 2164-2173.

Nasreddine, Z. S., Phillips, N. A., Bédirian, V., Charbonneau, S., Whitehead, V., Collin, I., ... Chertkow, H. (2005). The Montreal Cognitive Assessment, MoCA: A brief screening tool for mild cognitive impairment. Journal of the American Geriatrics Society, 53(4), 695-699.

Nyberg, L., Salami, A., Andersson, M., Eriksson, J., Kalpouzos, G., Kauppi, K., ... Nilsson, L.-G. (2010). Longitudinal evidence for diminished frontal cortex function in aging. Proceedings of the National Academy of Sciences, 107(52), 22682-22686.

Peters, F., Villeneuve, S., \& Belleville, S. (2014). Predicting progression to dementia in elderly subjects with mild cognitive impairment using both cognitive and neuroimaging predictors. Journal of Alzheimer's Disease, 38(2), 307-318.

Pruessner, J. C., Köhler, S., Crane, J., Pruessner, M., Lord, C., Byrne, A. .. Evans, A. C. (2002). Volumetry of temporopolar, perirhinal, entorhinal and parahippocampal cortex from high-resolution MR images: Considering the variability of the collateral sulcus. Cerebral Cortex, 12 (12), 1342-1353.

Pruessner, J., Li, L., Serles, W., Pruessner, M., Collins, D., Kabani, N., .. Evans, A. (2000). Volumetry of hippocampus and amygdala with highresolution MRI and three-dimensional analysis software: Minimizing the discrepancies between laboratories. Cerebral Cortex, 10(4), 433-442.

Rabin, L. A., Paré, N., Saykin, A. J., Brown, M. J., Wishart, H. A., Flashman, L. A., \& Santulli, R. B. (2009). Differential memory test sensitivity for diagnosing amnestic mild cognitive impairment and predicting conversion to Alzheimer's disease. Aging, Neuropsychology, and Cognition, 16(3), 357-376.

Ranganath, C., Yonelinas, A. P., Cohen, M. X., Dy, C. J., Tom, S. M., \& D'esposito, M. (2004). Dissociable correlates of recollection and familiarity within the medial temporal lobes. Neuropsychologia, 42(1), 2-13.

Raz, N., Lindenberger, U., Rodrigue, K. M., Kennedy, K. M., Head, D. Williamson, A., ... Acker, J. D. (2005). Regional brain changes in aging healthy adults: General trends, individual differences and modifiers. Cerebral Cortex, 15(11), 1676-1689.

Raz, N., Rodrigue, K., Head, D., Kennedy, K., \& Acker, J. (2004). Differential aging of the medial temporal lobe a study of a five-year change. Neurology, 62(3), 433-438.

Reitan, R. M. (1992). Trail Making Test: Manual for administration and scoring. Tucson, AZ: Reitan Neuropsychology Laboratory.

Scahill, R. I., Frost, C., Jenkins, R., Whitwell, J. L., Rossor, M. N., \& Fox, N. C. (2003). A longitudinal study of brain volume changes in normal aging using serial registered magnetic resonance imaging. Archives of Neurology, 60(7), 989-994.

Schacter, D. L., Koutstaal, W., \& Norman, K. A. (1997). False memories and aging. Trends in cognitive sciences, 1(6), 229-236. 
Schacter, D. L., Israel, L., \& Racine, C. (1999). Suppressing false recognition in younger and older adults: The distinctiveness heuristic. Journal of Memory and Language, 40(1), 1-24.

Schmidt, M. (1996). Rey auditory verbal learning test: A handbook. Los Angeles, CA: Western Psychological Services.

Schoemaker, D., Gauthier, S., \& Pruessner, J. C. (2014). Recollection and familiarity in aging individuals with mild cognitive impairment and Alzheimer's disease: A literature review. Neuropsychology Review, 24 (3), 313-331.

Schonfield, D., \& Robertson, B. A. (1966). Memory storage and aging. Canadian Journal of Psychology, 20(2), 228-236.

Simons, J. S., Verfaellie, M., Galton, C. J., Miller, B. L., Hodges, J. R., \& Graham, K. S. (2002). Recollection-based memory in frontotemporal dementia: Implications for theories of long-term memory. Brain, 125 (11), 2523-2536.

Sled, J. G., Zijdenbos, A. P., \& Evans, A. C. (1998). A nonparametric method for automatic correction of intensity nonuniformity in MRI data. IEEE Transactions on Medical Imaging, 17(1), 87-97.

Smith, A. (1973). Symbol digit modalities test. Los Angeles, CA: Western Psychological Services.

Smith, C. N., Wixted, J. T., \& Squire, L. R. (2011). The hippocampus supports both recollection and familiarity when memories are strong. The Journal of Neuroscience, 31(44), 15693-15702.

Smith, J. A., \& Knight, R. G. (2002). Memory processing in Alzheimer's disease. Neuropsychologia, 40(6), 666-682.

Shimamura, A. P., Janowsky, J. S., \& Squire, L. R. (1990). Memory for the temporal order of events in patients with frontal lobe lesions and amnesic patients. Neuropsychologia, 28(8), 803-813.

Sullivan Giovanello, K., Schnyer, D. M., \& Verfaellie, M. (2004). A critical role for the anterior hippocampus in relational memory: Evidence from an fMRI study comparing associative and item recognition. Hippocampus, $14(1), 5-8$.

Tombaugh, T. N., Kozak, J., \& Rees, L. (1999). Normative data stratified by age and education for two measures of verbal fluency: FAS and animal naming. Archives of Clinical Neuropsychology, 14(2), 167-177.

Vann, S. D., Tsivilis, D., Denby, C. E., Quamme, J. R., Yonelinas, A. P., Aggleton, J. P., ... Mayes, A. R. (2009). Impaired recollection but spared familiarity in patients with extended hippocampal system damage revealed by 3 convergent methods. Proceedings of the National Academy of Sciences, 106(13), 5442-5447.
Wechsler, D. (1997). WAIS-III: Administration and scoring manual: Wechsler adult intelligence scale. San Antonio, TX: Psychological Corporation.

Wechsler, D., \& Hsiao-Pin, C. (2011). WASI-II: Wechsler abbreviated scale of intelligence. San Antonio, TX: Pearson.

West, R. L. (1996). An application of prefrontal cortex function theory to cognitive aging. Psychological Bulletin, 120(2), 272-292.

Whitwell, J. L., Crum, W. R., Watt, H. C., \& Fox, N. C. (2001). Normalization of cerebral volumes by use of intracranial volume: Implications for longitudinal quantitative MR imaging. American Journal of Neuroradiology, 22(8), 1483-1489.

Wilding, E. L., \& Rugg, M. D. (1997). Event-related potentials and the recognition memory exclusion task. Neuropsychologia, 35(2), 119-128.

Wolk, D. A., Dunfee, K. L., Dickerson, B. C., Aizenstein, H. J., \& DeKosky, S. T. (2011). A medial temporal lobe division of labor: Insights from memory in aging and early Alzheimer disease. Hippocampus, 21(5), 461-466.

Yesavage, Jerome A, Brink, Terence L, Rose, Terence L, Lum, Owen, Huang, Virginia, Adey, Michael, \& Leirer, Von Otto. (1983). Development and validation of a geriatric depression screening scale: a preliminary report. Journal of psychiatric research, 17(1), 37-49.

Yonelinas, A. P. (2002). The nature of recollection and familiarity: A review of 30 years of research. Journal of Memory and Language, 46 (3), 441-517.

Yonelinas, A. P., Hopfinger, J. B., Buonocore, M. H., Kroll, N. E. A., \& Baynes, K. (2001). Hippocampal, parahippocampal and occipitaltemporal contributions to associative and item recognition memory: An fMRI study. Neuroreport, 12(2), 359-363.

Yonelinas, A. P., Widaman, K., Mungas, D., Reed, B., Weiner, M. W., \& Chui, H. C. (2007). Memory in the aging brain: Doubly dissociating the contribution of the hippocampus and entorhinal cortex. Hippocampus, 17(11), 1134-1140. 